\title{
Análise Comparativa da Infiltração Apical entre quatro Diferentes Materiais Retro-obturadores
}

\author{
Comparative Analysis of Apical Leakage Among four Differents Retrofilling Materials
}

\section{George Táccio de Miranda Candeiro ${ }^{1}$, Janaína de Oliveira Braga², Marco Antônio Húngaro Duarte ${ }^{3}$, llan Sampaio do Vale}

\section{Abstract}

The aim of this study was to compare the apical sealing ability between the white MTA, gray MTA, Super EBA and Sealapex/Zinc Oxide association when used as root-end filling materials. Forty four maxillary central incisors extracted were distributed in four groups with 10 specimens each and two control groups with two specimens each. The teeth had their crowns sectioned and their canals instrumented by step-back technique to \#60 file, using constant irrigation of $2.5 \%$ sodium hypochlorite. Roots were filled with guttapercha and EndoFill sealer by Tagger's hybrid technique. The apical $3 \mathrm{~mm}$ of each root was resected, and 3-mm deep root-end cavity preparations were made. The root-end cavities were filled with white MTA, gray MTA, Super EBA and Sealapex/ZO. The roots were immersed in Rhodamine $B$ and kept in an oven for 72 hours at $37^{\circ} \mathrm{C}$. A longitudinal section was performed to verify using Image Tool the dye penetration. Statistical analysis showed that the group sealed with Super EBA showed lower apical leakage than the other groups, showing significant difference $(p<0.05)$ only in relation to white MTA and gray MTA. It was concluded that the Super EBA and the Sealapex/ZO association showed adequate apical seal in retrofillings.

Keywords: Endodontics; Dental Materials; Dental leakage.

\section{Resumo}

O objetivo deste trabalho foi comparar ex vivo a capacidade seladora apical entre o MTA branco, o MTA cinza, o Super EBA e a associação Sealapex/Óxido de Zinco quando usados como materiais retro-obturadores. Foram divididos 44 incisivos centrais superiores humanos extraídos em quatro grupos experimentais com 10 espécimes cada e dois grupos controles com 2 espécimes cada. Os dentes tiveram as coroas seccionadas e seus canais instrumentados pela técnica escalonada até o instrumento \#60, usando irrigação constante com hipoclorito de sódio $2,5 \%$. As raízes foram obturadas com cones de guta-percha e cimento endodôntico EndoFill, pela técnica híbrida de Tagger. Os $3 \mathrm{~mm}$ apicais de cada raiz foram seccionados e foram feitas cavidades apicais com $3 \mathrm{~mm}$ de profundidade. As cavidades apicais foram preenchidas com os materiais MTA branco, MTA cinza, Super EBA e Sealapex/OZ. As raízes foram imersas no corante Rodamina B e mantidas numa estufa por 72 horas, à temperatura de $37^{\circ} \mathrm{C}$. Uma secção longitudinal foi realizada, a fim de verificar usando o programa Image Tool a penetração do corante. A análise estatística mostrou que o grupo selado com o Super EBA exibiu menores infiltrações apicais do que os demais grupos experimentais, apresentando diferença significante $(p<0,05)$ apenas em relação ao MTA branco e o MTA cinza. Concluiu-se que o Super EBA e a associação Sealapex/OZ apresentaram selamento apical adequado em retro-obturações.

Palavras-chave: Endodontia; Materiais odontológicos; Infiltração dentária.
${ }^{1}$ Especialista em Endodontia pela Universidade Federal do Ceará. Mestre em Clínica Odontológica, área temática Endodontia, pela Universidade Federal do Ceará. Doutorando em Endodontia pela Faculdade de Odontologia da Universidade de São Paulo.

${ }^{2}$ Especialista em Endodontia pela Universidade Federal do Ceará. Mestranda em Geriatria pela Universidade de Lisboa. Estagiária docente da disciplina de Endodontia da Universidade de Lisboa.

${ }^{3}$ Mestre e Doutor em Endodontia pela Faculdade de Odontologia de Bauru da Universidade de São Paulo. Professor do curso de Odontologia da Faculdade de Odontologia de Bauru da Universidade de São Paulo.

${ }^{4}$ Especialista, Mestre e Doutor em Endodontia pela Faculdade de Odontologia de Bauru da Universidade de São Paulo. Professor do curso de Odontologia da Universidade Federal do Ceará

Correspondência: George Táccio de Miranda Candeiro

Endereço: Av. Desembargador Moreira, 2660 - CEP 60135-690, Fortaleza - CE, Brasil

E-mail: georgecandeiro@hotmail.com

Data de Submissão: 20/01/2010

Data de Aceite: 14/05/2010

\section{Introdução}

A preservação dos dentes naturais é um dos principais desafios da odontologia moderna. No caso das periapicopatias, patógenos potenciais são eliminados pelo tratamento endodôntico e com subseqüente tratamento restaurador coronário. Contudo, em certos casos, não ocorre reparação tecidual, indicando a permanência de agentes nocivos dentro do sistema de canais radiculares. Se o retratamento endodôntico não for indicado, nem possível, a cirurgia parendodôntica é utilizada como última manobra para salvar o dente afetado (CANDEIRO et al., 2008).

Atualmente, existem diversas modalidades cirúrgicas que visam solucionar os problemas criados pelo tratamento endodôntico ou não solucionáveis por ele. Entretanto, as cirurgias parendodônticas podem fracassar devido ao selamento incompleto do sistema de canais radiculares.

Entre as modalidades de cirurgia parendodôntica, a apicetomia e a obturação retrógrada têm como objetivo preparar a região apical para receber um material capaz de promover o melhor selamento possível, dificultando a micro-infiltração e a recidiva da lesão. Para isso, há a necessidade de se confeccionar uma cavidade retrógrada adequada, com paredes regulares acompanhando o canal principal em profundidade suficiente para que o material retro-obturador seja capaz de vedá-la de modo satisfatório (WESTON; BARTOLD, 1999).

Um material ideal para ser utilizado em obturações retrógradas deve aderir às paredes da cavidade, promovendo o selamento do sistema de canais radiculares. Deve ser bem tolerado pelos tecidos periapicais, estimular o reparo, ser facilmente manipulado, ser radiopaco e estável dimensionalmente, não deve ser tóxico ou sofrer corrosão, não deve ser reabsorvido nem afetado pela umidade (GARTNER; DORN, 1990).

Diversos materiais têm sido sugeridos para 0 preenchimento da cavidade retrógrada, sendo os mais comumente empregados os cimentos de ionômero de vidro, o amálgama, os cimentos à base de óxido de zinco e eugenol e, mais recentemente, o Mineral Trióxido Agregado. O Super EBA é um cimento a base de óxido de zinco e eugenol modificado com ácido 
etoxi-benzóico, para melhorar a configuração com o tempo e aumentar a resistência da mistura, que demonstra alta resistência à compressão e à tração, $\mathrm{pH}$ neutro e baixa solubilidade, adere à estrutura dental mesmo em umidade, proporcionando uma boa cicatrização.

O Mineral Trióxido Agregado (MTA) tem sido investigado como um material de preenchimento em Endodontia, podendo ser utilizado em reabsorções dentárias internas e externas, perfurações, capeamento pulpar direto, apicigênese e apicificação, retro-obturações e obturações retrógradas (ALMEIDA; SOUSA JUNIOR; SANTOS, 2004). O MTA possui importantes propriedades físicas e biológicas, como adequada biocompatibilidade (TORABINEJAD et al., 1995), adaptação marginal às paredes de uma cavidade (PETERS; PETERS, 2002) e possibilidade de utilização em campo úmido (BUSATO et al., 1999).

Dentre os cimentos à base de hidróxido de cálcio destaca-se o Sealapex, por apresentar boas propriedades biológicas. O emprego do cimento Sealapex em obturações retrógradas é possível por meio do seu acréscimo ao óxido de zinco, proporcionando condições de condensação do material na cavidade retrógrada

O objetivo deste trabalho foi comparar ex vivo a capacidade seladora apical do MTA branco, MTA cinza, Super EBA e da associação entre o cimento endodôntico Sealapex e o pó de óxido de zinco em obturações retrógradas.

\section{Material e Método}

Este projeto foi, inicialmente, submetido e aprovado pelo Comitê de Ética em Pesquisa da Universidade Federal do Ceará, uma vez que os princípios éticos da Declaração de Helsinki (2000) e a Resolução CNS 196/96 foram obedecidos.

Foram selecionados para a pesquisa 44 dentes incisivos centrais superiores humanos extraídos, armazenados em solução de timol 0,01\%, que apresentavam somente uma raiz e um conduto, devendo também apresentar rizogênese completa com comprovação clínica e radiográfica. Esses dentes foram distribuídos em 4 grupos experimentais (I, II, III e IV) com 10 espécimes cada e dois grupos controle com dois espécimes cada.

Os grupos experimentais foram divididos de acordo com o tipo de material retro-obturador utilizado no preenchimento do retropreparo (tabela 1).

Tabela 1. Distribuição dos grupos experimentais de acordo com o material retro-obturador empregado.

\begin{tabular}{|c|c|c|}
\hline $\begin{array}{l}\text { Grupo } \\
(n=10)\end{array}$ & Material & Fabricante \\
\hline I & MTA® Branco & Ângelus, Londrina, PR. Brasil. \\
\hline ॥ & MTA® Cinza & Ângelus, Londrina, PR, Brasil. \\
\hline III & Super EBA® & $\begin{array}{c}\text { Bosworth Corporation, Skokie, IL, } \\
\text { EUA }\end{array}$ \\
\hline & Sealapex $\AA^{1}$ & $\begin{array}{c}{ }^{1} \text { Sybron Endo Corporation, } \\
\text { Orange, CA, EUA. }\end{array}$ \\
\hline IV & $\stackrel{+}{\text { Óxido de Zinco }}{ }^{2}$ & $\begin{array}{c}{ }^{2} \text { Odahcam Herpo Produtos } \\
\text { Dentários Ltda, Rio de Janeiro, } \\
\text { RJ, Brasil }\end{array}$ \\
\hline
\end{tabular}

Os dentes foram seccionados na junção amelocementária com disco de carborundum acoplado a um contra-ângulo e micromotor em baixa rotação com abundante refrigeração, a fim de se trabalhar somente com a porção radicular. Os canais foram instrumentados pela técnica escalonada até o instrumento \#60, tendo irrigação constante com $2 \mathrm{~mL}$ de solução de hipoclorito de sódio $2,5 \%$ a cada troca de instrumento. Após remoção de smear layer com EDTA $17 \%$ por 3 minutos, seguida pela irrigação com hipoclorito de sódio $2,5 \%$ e água destilada, os canais foram secos com pontas de papel absorvente. As raízes foram então obturadas com cones de guta-percha (Dentsply, Maillefer, Ballaigues, Suíça) e cimento endodôntico EndoFill (Dentsply, Maillefer, Ballaigues, Suíça), utilizando a técnica híbrida de Tagger.

Em seguida, os ápices foram cortados, com angulação de $90^{\circ}$ com o longo-eixo radicular, a $3 \mathrm{~mm}$ do ápice com broca Zecrya (Dentsply, Maillefer, Ballaigues, Suíça) em alta rotação e irrigação constante com soro fisiológico. As raízes foram completamente impermeabilizadas com duas camadas de esmalte de unha e uma camada de araldite, exceto a superfície apical correspondente à cavidade retrógrada.

Foram realizados os retro-preparos com $3 \mathrm{~mm}$ de profundidade, seguindo o longo-eixo radicular, utilizando-se a retroponta ultra-sônica S12D90 (Gnatus, Ribeirão Preto, São Paulo, Brasil) acoplada no aparelho Jet Sonic Plus (Gnatus, Ribeirão Preto, São Paulo, Brasil), sendo utilizada a potência máxima com abundante irrigação de soro fisiológico. As cavidades retrógradas foram preenchidas com os materiais usados de acordo com os grupos. Cada material foi misturado com uma espátula 24 em uma placa de vidro, a fim de se obter uma mistura homogênea de acordo com as recomendações dos fabricantes. No caso da associação Sealapex/OZ, a proporção de cada material foi baseada nos trabalhos de Tanomaru Filho et al. (1998) e Tanomaru Filho, Tanomaru e Faleiros (2004).

Nos grupos controle positivo e negativo, os espécimes tiveram os preparos apicais realizados semelhantemente aos grupos experimentais. Entretanto, no positivo não foi realizada nenhuma retro-obturação, a fim de se obter máxima infiltração de corante. Os espécimes do grupo controle negativo tiveram suas cavidades retrógradas preenchidas com MTA branco, seguido de completa impermeabilização externa com duas camadas de esmalte de unha e uma camada de araldite.

As raízes foram imersas no corante Rodamina $B$ à $0,2 \%$ e mantidas numa estufa por 72 horas, à temperatura de $37^{\circ} \mathrm{C}$. Após este período, as raízes foram lavadas em água corrente por 24 horas. Realizou-se cuidadoso desgaste longitudinal da porção apical radicular no sentido vestíbulo-palatino até a exposição do canal radicular e da retro-obturação, com finos discos de aço de carborundum em baixa rotação.

As raízes foram escaneadas e analisadas pelo programa de software Image Tool (UTHSCSA Dental Diagnostic Science, TX EUA), onde foram anotadas as médias das infiltrações nas faces vestibulares e linguais. Estas foram transformadas em escores $(0 \mathrm{a}$ 5), de acordo com a percentagem de infiltração do corante pela interface dente/material retro-obturador (tabela 2), conforme Candeiro, Duarte e Vale (2008). Os escores obtidos foram submetidos ao teste Kruskal-Wallis e comparações com Teste de Dunn, sendo considerado significante quando $p<0,05$.

Tabela 2. Escores adotados a partir das infiltrações apicais nas obturações retrógradas.

\begin{tabular}{cc}
\hline Escore & Infiltração Apical \\
\hline 0 & Ausência de infiltração (0\%) \\
1 & Infiltração $>0 \%$ e $\leq 25 \%$ \\
2 & Infiltração $>25 \%$ e $\leq 50 \%$ \\
3 & Infiltração $>50 \%$ e $\leq 75 \%$ \\
4 & Infiltração $>75 \%$ e $<100 \%$ \\
5 & $100 \%$ de infiltração \\
\hline
\end{tabular}

\section{Resultados}

Foi observado que o grupo controle positivo apresentou as maiores infiltrações, uma vez que o corante penetrou completamente nas cavidades retrógradas. Já os espécimes do grupo controle negativo não apresentaram nenhuma infiltração. 
Após análise estatística pelo teste não-paramétrico de Kruskal-Wallis, e comparações pelo método de Dunn, verificou-se que houve diferença estatística $(p<0,05)$ entre os grupos experimentais (Figura 1). O Super EBA foi 0 material que apresentou o menor grau de infiltração apical, embora sem diferença significante $(p>0,05)$ apenas em relação à associação Sealapex/OZ (Tabela 3).

As médias dos escores das infiltrações apicais apresentadas por cada grupo estão na tabela 3.

Tabela 3. Média \pm desvio padrão dos escores de infiltração apical nos grupos experimentais. Letras diferentes indicam que houve diferença significante $(p<0,05)$.

\begin{tabular}{ccc}
$\begin{array}{c}\text { Grupo } \\
(\mathrm{n}=10)\end{array}$ & Material Utilizado & $\begin{array}{c}\text { Médias dos Escores } \pm \\
\text { Desvio-padrão }\end{array}$ \\
\hline I & MTA branco & $4,4 \pm 0,8433^{\mathrm{a}}$ \\
II & MTA cinza & $3,1 \pm 0,9944^{\mathrm{ab}}$ \\
III & Super EBA & $1,5 \pm 0,5270^{\mathrm{c}}$ \\
IV & Sealapex + Óxido de Zinco & $2,7 \pm 1,2520^{\mathrm{bc}}$ \\
\hline
\end{tabular}

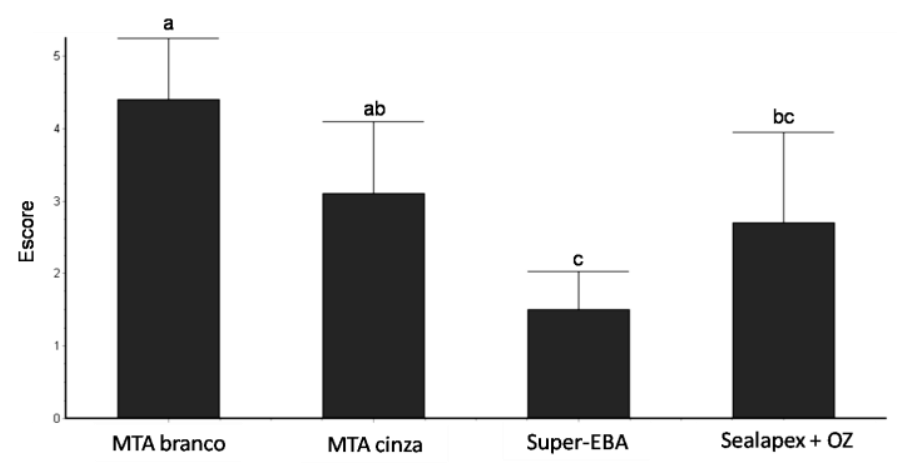

Figura 1. Infiltrações marginais em retro-obturações nos grupos experimentais.

Letras diferentes indicam que houve diferença significante $(p<0,05)$.

\section{Discussão}

A complexidade do sistema de canais radiculares, juntamente com a enorme quantidade de túbulos dentinários presentes constituem possíveis sítios capazes de abrigar bactérias com potencial patogênico suficiente para manter lesões periapicais, representando insucesso no tratamento endodôntico.

A região apical, por possuir a maior concentração de ramificações do sistema de canais radiculares, necessita de uma atenção especial durante a cirurgia parendodôntica, a fim de eliminar algum fator irritante local (HSU; KIM, 1997). Dessa forma, a apicetomia com $3 \mathrm{~mm}$ de extensão e angulação de $90^{\circ}$ com o longo-eixo radicular torna-se um importante procedimento, pois remove a região de delta apical, sendo esta a mais freqüente ramificação do sistema de canais radiculares (NICHOLLS, 1965; GAGLIANI; TASCHIERI; MOLINARI, 1998). A referida angulação é importante para não haver a exposição de muitos túbulos dentinários, que poderiam abrigar bactérias remanescentes, perpetuando lesões perriradiculares (GILHEANY; FIGDOR; TYAS, 1994).

Outro fator importante está na confecção do retro-preparo, que deve preferencialmente ser realizada com retro-pontas ultrasônicas, pois permitem realizar cavidades com profundidade adequada, seguindo o longo-eixo radicular, podendo ainda ser usadas em regiões de istmos (VON ARX; WALKER, 2000). Alguns autores não verificaram in vitro diferenças estatísticas entre retropreparos realizados com retro-pontas ultra-sônicas e com broca esférica (ASSIS et al., 2003; CANDEIRO; DUARTE; VALE, 2008) No entanto, clinicamente, Lange et al. (2007) observaram uma maior taxa de sucesso quando da utilização da técnica ultrassonoenergizada.

A obturação retrógrada constitui uma complementação ao retro-preparo, a fim de selar a cavidade formada, evitando a passagem de microrganismos para dentro do canal radicular, sendo importante para o processo de reparo apical. Assim, vários materiais retro-obturadores, como o MTA, o super EBA e o EBA, têm sido empregados na busca de um material ideal com propriedades físico-químicas e biológicas adequadas.

No presente estudo, comparou-se o selamento apical promovido por alguns materiais usados em obturação retrógrada, como o Mineral Trióxido Agregado (MTA) branco e cinza, o Super EBA e a associação entre o cimento endodôntico Sealapex e o Óxido de Zinco, empregando o corante Rodamina B como marcador de infiltração.

Os resultados do presente estudo mostraram que o Super EBA apresentou o melhor selamento marginal quando comparado aos outros materiais testados, porém com diferenças significantes apenas em relação ao MTA branco e ao MTA cinza. Já os resultados de Torabinejad et al. (1993), Pereira, Cenci e Demarco (2004) apontam para uma maior capacidade seladora do MTA em relação ao Super EBA com diferença estatisticamente significante, ao passo que Gonçalves e Bramante (2002), Gomes et al. (2003), Silva Neto et al. (2003), Pichardo et al. (2006) e Gomes et al. (2009) observaram selamentos similares entre os dois materiais. Isso pode ter relação com o menor tempo de presa do Super EBA em relação aos demais materiais, principalmente o MTA que possui tempo de presa superior a duas horas, segundo Torabinejad et al.(1995a).

Nos trabalhos de Leski e Pawlicka (2003) e de Pereira, Cenci e Demarco (2003) foi utilizado o corante azul de metileno, a fim de analisar o grau de infiltração marginal em retro-preparos, cujo cimento retro-obturador foi o MTA. Segundo Wu, Kontakoitis e Wesselink (1998), Tanomaru Filho, Figueiredo e Tanomaru (2005), o corante azul de metileno, em contato com MTA, sofre descoloração, uma vez que possui instabilidade na presença de alcalinidade do cálcio. Assim, o óxido de cálcio, contido no MTA, quando em contato com água, forma hidróxido de cálcio que acaba por descolorir o azul de metileno. Assim, pode-se inferir que os resultados de alguns trabalhos com a utilização do corante azul de metileno em contato com o MTA são questionáveis pois podem subestimar as reais infiltrações marginais neste material. Por isso, no presente trabalho, o corante utilizado foi a Rodamina B, conforme as pesquisas de Gonçalves e Bramante (2002), Silva Neto et al. (2003), Torabinejad, Watson e Pitt Ford (1993) e Valera et al. (2006).

Existem duas formas de apresentação do Mineral Trióxido Agregado, o cinza e o branco, embora ambos possuam composição química semelhantes, apresentando poucas diferenças, como a menor concentração de óxido férrico no MTA branco (ASGARY et al. 2005). Diante de uma escassez de trabalhos que comparassem $O$ selamento em cavidades retrógradas preenchidas com o MTA cinza e o MTA branco, no presente trabalho não foi verificada diferenças significativas entre os dois materiais, concordando com Ferris e Baumgartner (2004) e Mohammadi (2008), embora Storm et al. (2008) tenham verificado que o MTA cinza sofre expansão significantemente maior do que o MTA branco, apresentando a possibilidade de promover um melhor selamento por diminuir espaços na interface dentina/material.

Ghaziani e Sadeghi (2008) verificaram uma maio capacidade seladora do MTA branco em comparação ao MTA cinza, com diferença estatística significante, porém, a análise foi realizada pela penetração do corante azul de metileno, que conforme relatado pode conduzir a falsos resultados, não sendo portanto comparado aos resultados do presente trabalho.

Tem sido sugerida a utilização do cimento endodôntico Sealapex associado ao pó de óxido de zinco, formando uma 
mistura homogênea, capaz de ser condensado, podendo selar satisfatoriamente a cavidade retrógrada, além de possuir adequada biocompatibilidade tecidual (VALERA et al., 2005).

Entretanto, Tanomaru Filho, Tanomaru e Faleiros (2004) verificaram uma menor capacidade seladora da associação entre 0 cimento endodôntico Sealapex e o Óxido de Zinco do que o MTA, quando utilizado em perfurações de furca, porém neste trabalho o corante usado foi 0 azul de metileno, conduzindo resultados questionáveis, conforme anteriormente comentado. Os resultados do presente trabalho mostraram que o grupo em que foi utilizada a referida associação apresentou menores infiltrações marginais do que os grupos que utilizaram o Mineral Trióxido Agregado (branco e cinza), porém sem diferença estatística apenas em relação ao grupo selado com MTA cinza, concordando com Valera et al. (2006)

\section{Conclusões}

Diante da metodologia empregada concluiu-se que o Super EBA e a associação Sealapex/OZ apresentaram selamento apical adequado em retro-obturações, podendo ser opções como materiais de preenchimento apical.

\section{Referências}

ALMEIDA, T. S.; SOUSA JUNIOR., H. A.; SANTOS, W. A. G. MTA: suas propriedades físicas e biológicas. Robrac, Goiania, v. 13, n. 35, p.1-11, 2004.

ASGARY, S. et al. Chemical differences between white and gray mineral trioxide aggregate. J. Endod., Chicago, v. 31, no. 2, p. 101-103, Feb. 2005.

ASSIS, N.M.S.P. et al. Avaliação do selamento de ápices radiculares preparados com ultra-som e brocas e retro-obturados com diferentes materiais mediante infiltração marginal por corante.

R. Odontol. UNESP, Araçatuba, v. 32, n. 1, p.1-8, 2003.

BUSATO, A.L.S. et al. Agregado de trióxido mineral: indicações clínicas de um novo cimento dentário. JBC: J. Bras. Clin. Estet. Odontol., Curitiba, v. 3, n.18, p.32-34, dez. 1999.

CANDEIRO, G.T.M.; DUARTE, M.A.H.; VALE, I.S. Avaliação da infiltração apical utilizando diferentes retro-pontas ultra-sônicas e broca esférica. R. Odontol. Araçatuba, Araçatuba, v. 29, n. 1, p. 25-29, jan./jun. 2008

FERRIS, D.; BAUMGARTNER, J.C. Perforation repair comparing two types of mineral trioxide aggregate. J. Endod., Chicago, v. 30 , no. 6, p. 422-424, June 2004.

GAGLIANI, M.; TASCHIERI, S.; MOLINARI, R. Ultrasonic root-end preparation: influence of cutting angle on the apical seal. J. Endod., Chicago, v. 24, no. 11, p. 726-730, Nov. 1998.

GARTNER, A. H.; DORN, S. O. Retrograde filling material: a retrospective success-failure study of amalgam, EBA, and IRM. J. Endod., Chicago, v. 16, no. 4, p. 391-393, Apr. 1990.

GHAZIANI, P.; SADEGHI, G. An In Vitro Comparison of Apical Leakage of Biocalex, White MTA, Gray MTA, and Amalgam as Root-End Fillings. J. Dent., Tehran, Iran, v. 5, no. 3, p. 131-135, 2008

GILHEANY, P.A. ; FIGDOR, D.; TYAS, M.J. Apical dentin permeability and microleakage associated with root end resection and retrograde filling. J. Endod., Baltimore, v. 20, no. 1, p. 22-26, Jan. 1994
GOMES, A.P.M. et al. Avaliação do selamento de retro-obturações realizadas com super-EBA e MTA após preparo cavitário com ultrasom ou brocas. Ci. Odontol. Bras., São José dos Campos, v. 6, n. 2, p. 20-28, abr./jun. 2003.

GOMES C.C. et al. Análise da adaptação marginal de materiais retrobturadores. Pesq. Bras. Odontoped. Clin. Integr., João Pessoa, v. 9, n. 1, p. 31-35, jan./abr. 2009.

GONÇALVES, S. B.; BRAMANTE, C. M. Avaliação in vitro da capacidade seladora do super-eba e do mta em quatro técnicas de obturação retrógrada. R. Fac. Odontol. Bauru, Bauru, v. 10, n.3, p. 170-178, 2002

HSU, Y.Y.; KIM, S. The resected root surface. The issue of canal isthmuses. Dent. Clin. North Am., Philadelphia, v. 41, no. 4, p. 529-540, July 1997.

LANGE, J.; PUTTERS, T.; BASS, E.M.; van INGREN, J.M. Ultrasonic root-end preparation in apical surgery: a prospective randomized study. Oral Surg. Oral Med. Oral Pathol. Oral Radiol. Endod., St. Louis, v. 104, no. 6, p. 841-845, Dec. 2007.

LESKI, M.; PAWLICKA, $\mathrm{H}$. The effect of ultrasonic and bur root-end preparation on MTA root-end fillings. Int. Endod. J., Oxford, v. 36, no. 12, p. 942, Dec. 2003. Trabalho apresentado no European Society of Endodontology $11^{\text {th }}$ Biennial Congress, Athens, 2003.

MOHAMMADI, Z. Sealing ability of MTA cements as orthograde root filling materials. Pesq. Bras. Odontoped. Clin. Integr., João Pessoa, v. 8, n. 3, p. 267-270, set./dez. 2008.

NICHOLLS, E. The role of surgery in endodontics. Brit. Dent. J., London, v.18, no. 2, p. 59-71, Jan. 1965.

PEREIRA, C.L.; CENCI, M.S.; DEMARCO, F.F. Sealing ability of MTA, super EBA, vitremer and amalgam as root-end filling materials. Braz. Oral Res., São Paulo, v. 18, n. 4, p. 317-321, out./dez. 2004.

PETERS, C.I.; PETERS, O.A. Occlusal loading of eba and mta rootend fillings in a computer-controlled-masticator: a scanning electron microscopic study. Int. Endod. J., Oxford, v.35, no.1, p.22-29, Jan. 2002.

PICHARDO, M.R. et al. Apical leakage of root-end placed superEBA, MTA, and geristore restorations in human teeth previously stored in $10 \%$ formalin. J. Endod., Baltimore, v. 32 , no. 10, p. 956-959, Oct. 2006.

SILVA NETO, U.X. et al. Infiltração marginal em obturações retrógradas realizadas com ProRoot-MTA, MTA-Angelus e SuperEBA. JBE: J. Bras. Endod., Curitiba, v. 4, n.13, p.149-152, abr./jun. 2003.

STORM, B. et al. Setting expansion of gray and white mineral trioxide aggregate and Portland cement. J. Endod., Baltimore, v. 34, no. 1, p. 80-82, Jan. 2008.

TANOMARU FILHO, M. et al. Capacidade seladora de diferentes cimentos endodônticos em obturações retrógradas. $\mathbf{R}$. Fac Odontol. Lins, Lins, v.11, n.1, p.58-61, jan./jun. 1998.

TANOMARU FILHO, M.; TANOMARU, J.M.G.; FALEIROS, F.C.B. Capacidade seladora e adaptação de materiais utilizados em perfurações de furca. R. Fac. Odontol. Lins, Lins, v.16, n. 2, p.1924, jul./dez. 2004

TANOMARU FILHO, M.; FIGUEIREDO, F.A.; TANOMARU, J.M.G. Effects of differents dye solutions on the evaluation of the sealing 
ability of mineral trioxide aggregate. Braz. Oral Res., São Paulo, v. 19, n. 2, p. 119-122, abr./jun. 2005.

TORABINEJAD, $M$. et al. Citotoxicity of four root-end fillings materials. J. Endod., Baltimore, v. 21, no.10, p.489-492, Oct. 1995.

TORABINEJAD, M. et al. Physical and chemical properties of a new root-end filling material. J. Endod., Baltimore, v. 21, no.7, p.349353, July 1995a.

TORABINEJAD, M.; WATSON, T. F.; PITT FORD, T. R. Sealing ability of a mineral trioxide aggregate as a root end filling material. J. Endod., Baltimore, v.19, no.12, p.591-595, Dec. 1993.

VALERA, M.C. et al. Avaliação da compatibilidade biológica do cimento Sealapex ${ }^{\circledR}$ e deste cimento acrescido de iodofórmio ou óxido de zinco. Ci. Odontol. Bras., São José dos Campos, v. 8, n. 4, p. 29-38, out./dez 2005.

VALERA, M.C. et al. In vitro evaluation of apical microleakage using different root-end filling materials. J. Appl. Oral Sci., Bauru, v. 14, no. 1, p. 49-52, Jan./Feb. 2006.

VON ARX, T.; WALKER, W. A. Microsurgical instruments for rootend preparation following apicoectomy: a literature review. Endod. Dent. Traumat., Copenhagen, v. 16, no. 2, p. 47-62, Apr. 2000.

WESTON, G.D.; BARTOLD, P.M. A scanning electron microscopic evalution of root surfaces and the gutta-percha interface following root-end resection in vitro. Int. Endod. J., Oxford, v. 32, no. 6, p. 450-458, Nov. 1999.

WU, M.K. ; KONTAKOITIS, P.R. ; WESSELINK, P.R. Decoloration of $1 \%$ methylene blue solution in contact with dental filling materials. J. Dent., Bristol, v. 26, no. 7, p. 585-589, Sept.1998. 